\title{
Formation postgraduée en économie de la santé
}

L'économie de la santé et l'éthique font certes partie intégrante de chaque programme de formation postgraduée, mais pour les établissements de formation, elles ne sont pas faciles à enseigner car l'expérience fait défaut. Un programme modulaire de formation en ligne (e-learning) en économie de la santé permet à ces établissements d'offrir aux médecins en formation postgraduée un cours de haute qualité moyennant une charge administrative supplémentaire minime. Grâce au téléapprentissage, les médecins-assistants disposent d'une offre de cours compatible avec leurs horaires de travail, tout en pouvant tirer parti des temps morts ou des moments d'attente pour leur formation postgraduée.

\author{
Marc Otto ${ }^{a}$, Harry Telser ${ }^{b}$, \\ Max Giger \\ a Dr, pnn ag, Zurich \\ b Dr, Polynomics AG, Olten \\ c Dr, président de la CFPC, FMH
}

\section{Situation actuelle}

Le 31 mai 2005, le Département fédéral de l'intérieur (DFI) a accrédité les 44 programmes de formation postgraduée existants pour sept nouvelles années. Cette accréditation était liée, pour la FMH, à l'obligation d'inclure l'éthique et l'économie de la santé dans tous les programmes.

Pour les établissements de formation postgraduée, il convient maintenant de mettre en œuvre les exigences concernant l'éthique et l'économie de la santé stipulées au chiffre 5 des programmes de formation. Il s'agit, en l'occurrence, de donner un enseignement pratique sur la gestion indépendante des problèmes éthiques et d'économie de santé survenant dans la prise en charge de personnes malades et en bonne santé dans des situations typiques de la discipline concernée. Les médecins-assistants doivent acquérir la capacité d'appliquer, de manière autonome, des instruments qui facilitent les prises de décisions éthiques. Il leur faut aussi savoir gérer les problèmes économiques en utilisant de façon optimale les moyens à disposition et en tenant compte des bases légales, ce qui nécessite un enseignement explicite, basé sur des problèmes quotidiens concrets rencontrés dans la prise en charge des patients.

L'économie de la santé repose sur une manière de penser étrangère à de nombreux médecins. On la réduit souvent à des mesures de baisse des coûts, et donc à une toute petite partie de ce qu'elle est vraiment. De ce fait, une offre de cours en économie de la santé doit avant tout combattre les préjugés et transmettre correctement les notions nécessaires. L'économie de la santé étant une discipline théorique, basée avant tout sur des modèles, elle se prête tout particulièrement à une formation en ligne (e-learning).

\section{Idée à la base du projet}

En partant de ces prémisses, l'idée a été développée de remettre aux établissements de formation un instrument leur permettant d'offrir aux médecins-assistants un cours de formation postgraduée de haute qualité en économie de la santé. Ce cours devait posséder une structure modulaire, de façon à pouvoir accompagner et compléter, le cas échéant, des cours déjà existants. Son utilisation à l'hôpital ne devait entraîner qu'une charge administrative minimale. De plus, il convenait que les horaires de cours soient compatibles avec les heures de travail, de façon à ce que les médecins-assistants ne doivent pas «sacrifier» des jours de vacances pour cette formation, mais puissent au contraire utiliser les temps morts ou les moments d'attente pour s'y consacrer.

Pour éviter les conflits d'intérêts potentiels et accorder aux auteurs du cours une marge de liberté maximale, on a exclu tout financement préalable externe pour couvrir les coûts de développement du projet. En outre, on a institué un conseil spécialisé chargé de décider des contenus de manière définitive. Le cadre économique étant posé, il a fallu commencer par développer un module de base, dans l'idée que si un nombre suffisant de cours était «vendu», les moyens financiers ainsi obtenus permettraient de développer d'autres modules et de construire, pas à pas, une formation agencée selon le système modulaire. 


\section{Déroulement du projet}

Le système modulaire du cours a été défini conjointement avec le Dr Harry Telser, premier auteur: un module de base introduit les notions fondamentales et donne une première vue d'ensemble. Un catalogue de thèmes a été établi en vue d'un élargissement des connaissances, catalogue qui pourra être affiné une fois les premiers clients parvenus à ce stade.

En été 2007, le premier module a été rédigé en l'espace de trois mois par le Dr Telser et affiné dans le cadre d'une revue par des pairs, menée avec le Dr Berchtold, privat-docent, qui a accompagné le développement du projet du point de vue médical. Après avoir été évalué par un groupe de pairs à l'Hôpital Triemli, de Zurich, le module de base a été à nouveau révisé, puis soumis pour accréditation à la Fédération des médecins suisses (FMH), qui lui a attribué le label «Approuvé par la FMH» ainsi que deux crédits.

Entre-temps, cet enseignement en ligne a déjà été introduit avec succès dans différents établissements de formation postgraduée de Suisse alémanique.

\section{Le module de base}

Le module de base est téléchargeable sous www.pnn.ch/goek. Il est constitué de quatre cours, d'un contrôle des connaissances sous forme de jeu et d'un examen final. Les cours donnés disposent, outre de textes et d'illustrations, d'un glossaire, d'une bibliographie et de questions d'exercice intégrées. Le contrôle des connaissances est basé sur un jeu composé de questions et réponses à choix multiple, qui peuvent immédiatement être évaluées et comprennent un lien direct vers les cours en vue d'obtenir, sur demande, de l'aide pour répondre à la question posée. Le jeu par questions-réponses ainsi que l'examen sont automatiquement évalués, corrigés et commentés.

Le premier module (de base) propose une introduction aux notions fondamentales de l'économie de la santé. Le premier cours traite de notions telles que l'asymétrie de l'information, la théorie des mandats («agency theory»), les relations «principal-agent» dans le système de santé suisse (médecin-patient-assurance), alors que le deuxième aborde les effets de la protection d'assurance, le «risque moral» et les solutions permettant d'atténuer les effets de systèmes d'incitation problématiques. Le troisième cours propose une introduction à l'évaluation des questions d'économie de la santé, en particulier aux analyses de minimisation et de rentabilité des coûts ainsi qu'aux analyses coûts-efficacité et coûts-utilité. Quant au quatrième cours, il consiste en une répétition générale basée sur l'exemple de la prévention.

\section{Evaluation}

L'évaluation a été effectuée par le Département de médecine interne de l'Hôpital Triemli à Zurich. Pour l'enseignement en ligne, une fonctionnalité permettant de saisir des commentaires dans le contexte d'apprentissage avait été aménagée sur chaque page-écran. En outre, un formulaire d'évaluation standardisé avait été ajouté au module de façon à pouvoir évaluer ce dernier après l'avoir terminé. Ce formulaire comprenait douze questions, dont dix sous forme de réponses à choix multiple et deux invitant à rédiger librement un texte. Les questions se référaient au cours luimême ainsi qu'à des aspects spécifiques de l'enseignement en ligne.

Le groupe de pairs avait été recruté par le médecin-chef du département et informé par courriel directement par l'institut pnn ag. Ce groupe disposait de 14 jours pour tester le module, tout en ayant la liberté de décider dans quel ordre il allait le faire.

Le groupe comprenait treize médecins-assistants (six hommes et sept femmes), dont neuf (5 h, 4 f) ont rempli le formulaire d'évaluation.

Le temps de traitement médian du module s'est élevé à une heure 33 minutes, alors que les pages-écrans ont été consultées 180 fois en moyenne.

Des commentaires relevant quelques fautes d'orthographes et certaines inconsistances mineures entre texte et image ont été apposés en regard de diverses pages-écrans. Deux questions appelant un complément de contenu ont aussi été posées.

Le dépouillement des formulaires d'évaluation a mis en évidence un intérêt pour le thème de l'économie de la santé: bien que celui-ci soit qualifié $\mathrm{d}^{\prime}$ «assez peu relevant» par une majorité des participants à l'évaluation (6/9), le groupe de pairs considère que l'économie de la santé est un thème important (6/9). L'introduction d'une formation à distance a reçu un accueil favorable (3/9 très bien; $6 / 9$ bien) qui se reflète aussi dans l'évaluation des divers aspects du cours. Le groupe de pairs estime (5/9) qu'il serait très utile d'offrir certaines parties de la formation postgraduée médicale sous forme de téléapprentissage.

Le test effectué révèle que le cours en ligne a été bien accueilli par le groupe de pairs. L'économie de la santé est considérée par les assistants comme un thème important, trop peu abordé pendant la formation postgraduée. 


\section{Utilisation à I'hôpital}

Lorsqu'un établissement de formation postgraduée commande le cours de base en économie de la santé, il reçoit un certain nombre de documents PDF (PDF d'instruction aux utilisateurs). Ces documents sont un mode d'emploi expliquant non seulement que l'économie de la santé fait partie intégrante de tout cursus de formation postgraduée, mais aussi comment s'inscrire au cours, comment l'effectuer et faire usage du service d'assistance téléphonique aux utilisateurs. Le code d'accès, un nombre aléatoire relativement long permettant de suivre le cours aux conditions convenues avec l'établissement de formation postgraduée, figure également dans cette documentation. Lors de son premier accès au cours, chaque participant ou participante saisit ses données personnelles en vue de l'établissement d'une attestation de participation personnalisée à son intention. Ces instructions PDF sont par ailleurs adaptées à chaque établissement de formation et contiennent, outre le logo de celuici, un texte d'introduction spécifique rédigé par exemple par le médecin-chef de la clinique. De plus, les établissements peuvent fixer les conditions de participation au cours. A ce jour, la plupart d'entre eux ont décidé d'accorder un rabais de $100 \%$ à leurs membres, ce qui signifie que l'employeur prend les coûts à sa charge et offre à ses collaborateurs et collaboratrices un accès gratuit au cours.

Ce mode de faire, basé sur des instructions en format PDF, permet d'éviter une fastidieuse procédure d'inscription et de mutation et facilite aussi la distribution de l'accès au cours au sein de chaque hôpital.

\section{Evaluation du cours sous I'aspect économique}

La finance de participation au cours se monte à 150 francs, des rabais de quantite étant accordés aux établissements de formation de grande taille. Ces émoluments ne permettent pas seulement d'amortir les coûts de développement du cours, mais aussi les frais d'exploitation tels que la maintenance du serveur ou l'assistance aux utilisateurs. Pour presque tous les établissements de formation postgraduée, le recours à la formation en ligne se révèle plus économique que l'engage- ment d'un conférencier, car un programme de téléapprentissage peut être effectué quand il n'y a rien d'autre à faire, alors qu'une conférence a lieu lorsqu'un maximum de gens est présent dans l'institution, à savoir pendant les heures de travail régulières. En effet, pour l'organisation d'une session de formation postgraduée, il faut compter non seulement les honoraires de l'intervenant, mais également les coûts du temps de travail que les auditeurs passent dans la salle de conférences. Si les honoraires de l'intervenant se montent à 1500 francs, il faudrait au moins 21 assistants en formation postgraduée suivant l'exposé du début à la fin pour que le cours en auditoire se révèle plus avantageux que le cours en ligne (sans rabais de quantité). Et si l'on tient compte des coûts d'organisation et d'utilisation de la salle, le nombre de participants devrait être plus élevé encore.

\section{Perspectives}

Le module de base «Les fondements de l'économie de la santé» a d'ores et déjà été traduit en français et, après avoir été expertisé par un pair francophone, il se trouve en phase d'évaluation au Département de gynécologie et obstétrique du Centre Hospitalier Universitaire Vaudois (CHUV) à Lausanne.

En outre, des travaux sont en cours visant d'une part à combiner le module de base avec des cours existants proposés sous forme traditionnelle et, d'autre part, à développer ce cours modulaire en y ajoutant les contributions de nouveaux auteurs.

La combinaison d'une formation en ligne avec des périodes d'enseignement traditionnel («blended learning») permettrait par exemple d'acquérir les bases nécessaires par le biais du téléapprentissage, puis de les discuter et de les approfondir à l'aide d'exemples concrets dans le cadre d'un séminaire. Ceci représenterait non seulement un gain de temps, mais aussi une plusvalue pour le séminaire dont tous les participants disposeraient de connaissances préalables semblables.

Même si elle pose des exigences plus élevées, une formation en ligne semblable est actuellement à l'étude pour le domaine de l'éthique, lequel fait aussi partie intégrante de chaque programme de formation postgraduée. 